\title{
EDGE-ANCHORED MODE-MATCHED MICROMACHINED GYROSCOPIC DISK RESONATOR
}

\author{
Xudong Zou ${ }^{1,2}$, Chun Zhao and Ashwin A. Seshia ${ }^{1 *}$ \\ ${ }^{1}$ Nanoscience Centre, Department of Engineering, University of Cambridge, U.K. \\ ${ }^{2}$ Institute of Electronics, Chinese Academy of Sciences, CHINA
}

\begin{abstract}
This paper reports on a vacuum packaged circular disk gyroscopic resonator with T-shape anchors fabricated in a (100) single crystalline silicon substrate. This device topology simplifies the fabrication process as compared to previous approaches to realize center-anchored disk gyroscopes. Mode-matching of the trigonal modes of the disk is realized with open-loop characterization results demonstrating a Quality factor exceeding 1.5 million with an initial modal frequency split of $4.7 \mathrm{~Hz}$ and a natural frequency of approximately $0.976 \mathrm{MHz}$ (4.81 ppm split). An approach to effective mode matching of such devices is described.
\end{abstract}

\section{INTRODUCTION}

In recent years, the use of MEMS gyroscopes has expanded to address a growing number of applications, ranging from game controllers and mobile phones to digital cameras and wearable activity trackers [1]. The popularity of MEMS inertial sensors in commercial use is spurring research into the development of even more accurate devices which could satisfy high-end applications; particularly, a significant amount of effort is focused on the development of navigation-grade MEMS gyroscopes.

The device topology employed in many MEMS gyroscopes is a vibrating structure with axisymmetric features such as shells, rings or disks allowing for Coriolis coupling between near-degenerate vibrational modes [2]. More recently, gyroscopes that rely on bulk acoustic wave (BAW) vibration modes have been introduced [3, 4]. These devices operate at very high frequencies and have demonstrated substantial shock resistance and large bandwidth. Though mode-matching is desirable in such structures, there are always limitations due to fabrication tolerances, material anisotropy and practical anchoring arrangements employed. This often requires electrostatic tuning approaches to achieve mode-matching and the implementation of narrow capacitive gaps in high-aspect ratio structures for practical tuning at low voltages.

Conventionally, a single central anchor is considered to be an essential design feature for most BAW disk gyroscopes [3, 4], and fabrication of the central anchor normally relies on well-controlled sacrificial layer etching with etch holes required to be created within the disk for structural release. However, the addition of etch holes on the disk structure and the finite dimension of the central anchor can degrade the quality factor of the associated vibrational modes [4]. Further, fabrication tolerances associated with etch release might vary the dimension of the central anchor or misalign the position of the central anchor to break structural symmetry resulting in a further split between the frequencies of the drive and sense modes. This paper address some of these issues to demonstrate a disk gyroscope configuration using edge anchors on (100) single crystal silicon substrate achieving full high Q modematching at moderate tuning voltages. A further advantage of the edge-anchored design is that all critical dimensions of gyroscope structure are defined by one-step DRIE etching, which simplifies the fabrication process and facilitates tolerance control.

\section{DEVICE DESIGN}

\section{A) Edge-Anchored Disk Resonator}

The disk resonator is fabricated using (100) singlecrystal silicon (SCS) substrates due to their widely availability and potential for CMOS compatibility. However, the anisotropic material properties of the (100) SCS substrate, result in a splitting of the degeneracy of the two primary elliptic vibration modes making them unsuitable for gyroscope applications [3, 4]. As demonstrated in previous BAW disk gyroscopes with a central anchor, the secondary elliptic modes of disk with a trigonal mode shape (shown in Figure 1(a)) have nearly identical resonance frequencies and can allow for modematched operation. As shown in the COMSOL simulation, due to the anisotropic material properties of SCS, the secondary elliptical mode shape does not demonstrate the 30 degree rotational symmetry as for devices fabricated in an isotropic substrate but exhibits orthogonal symmetry with the symmetrical axis aligned to [100] and [010] directions. Figures 1 (b) and (c) show the secondary elliptical mode shape of the disk resonator replacing the central anchor with four identical ' $\mathrm{T}$ ' shape anchors connecting the disk to the substrate. The design introducing edge anchors on $\langle 110\rangle$ directions is preferred due to the smaller anchor displacement at resonance, reducing anchor loss, thus resulting in a higher quality factor of the resonator. Comparing to the central anchored disk resonator, the two degenerate trigonal modes of disk resonator with edge anchors shows mode shape distortion resulting in antinodes with maximum deflection not aligned to [100] and [010] directions but the resulting degenerate modes still preserves the orthogonal rotational symmetry between them.

\section{B) Mode Shape Control with T-shape Anchors}

Simulation results shown in Figure 1 shows that replacing the conventional central anchor with edge anchors in a disk resonator preserves the potential for degenerate mode operation but may potentially introduce mode-shape distortion. It is known that the antinodes and nodes of the two degenerate modes must be aligned to the surrounding electrodes for drive, sense and tuning to avoid cross-mode coupling and zero rate output. Hence, understanding mode-shape distortion in the above simulation results is critical for the placement and selection of the electrode locations around the disk circumference. 
Further inspection reveals that the mode-shape distortion is sensitive to the geometric dimensions of the T-shape edge anchors as illustrated by numerical modelling of the designs by FEA. In the 3D finite element model constructed for this purpose, the default diameter of disk is $6 \mathrm{~mm}$, the disk thickness is $40 \mu \mathrm{m}$, the horizontal beam length is $480 \mu \mathrm{m}$ and the vertical beam width is $60 \mu \mathrm{m}$. Figures 2 and 3 show the FEA (COMSOL) simulation results of the trigonal modes natural frequency and mode shape of disk resonator with the varying beam width and length of the T-shape edge anchors.

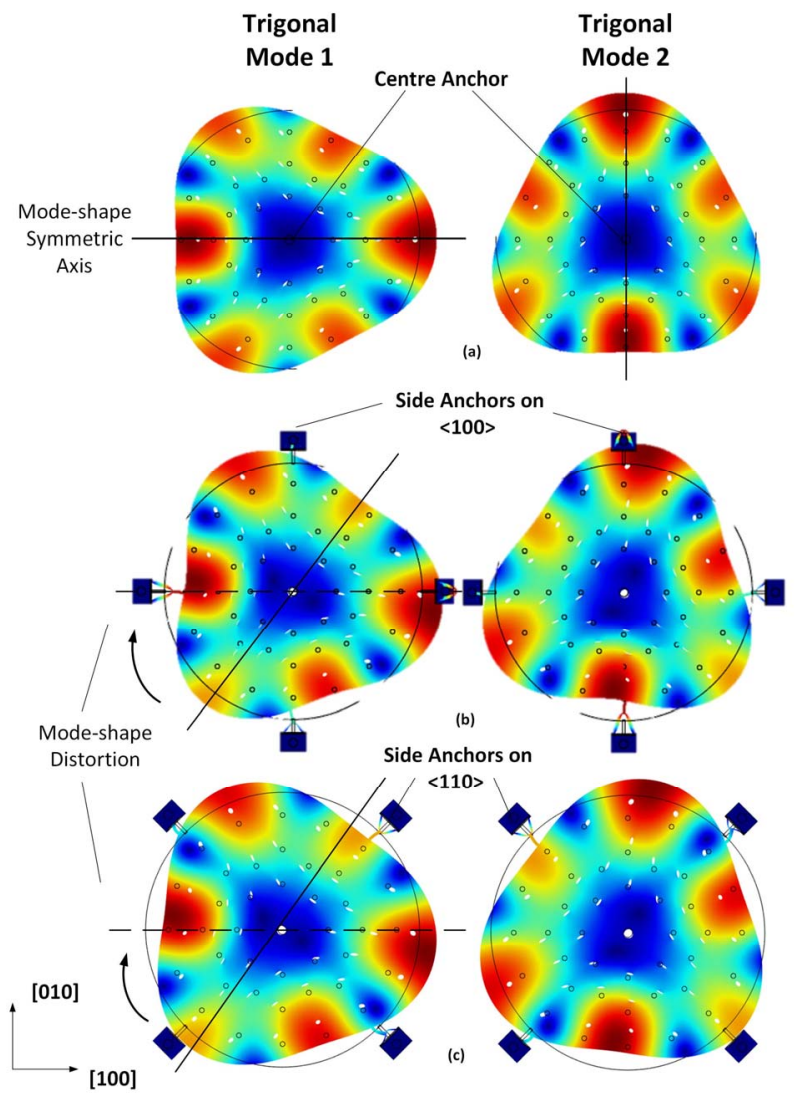

Figure 1: COMSOL simulation results of secondary elliptic degenerate modes shape for: (a) Disk resonator with single central anchor, (b) Disk resonator with edge anchors on $<100>$ directions, (c) Disk resonator with edge anchors on $<110>$ directions.

The simulation results indicate that varying the horizontal and vertical beam dimensions of the T-shape edge anchor can tune the axis of symmetry associated with the trigonal mode-shape from the $<100\rangle$ direction (distortion-free comparing to central anchor disk) to the $<110\rangle$ directions (distortion- maximum relative to the center-anchored disk). Considering the deflection of disk resonator in trigonal mode contains both a radial direction component and a tangential direction component, an intuitive explanation for such a tuning effect would be that: the radial and circumferential stiffness of the T-shape flexure structure, which is sensitive to the beam dimensions, may interact with the disk at the edges. If the ratio of radial and circumferential stiffness of the T-shape does not match to the ratio of local effective radial and circumferential stiffness of the disk at the edges, the radial direction component and tangential direction component of the disk resonator deflection at the connection point to the T-shape anchor is perturbed and thus results in mode-shape distortion. The simulation results shows that the variation of the T-shape beam dimensions result in a change of the mode resonance frequency as well, and thus the interaction between the design of the T-shape anchors and the disk resonator must be modelled.

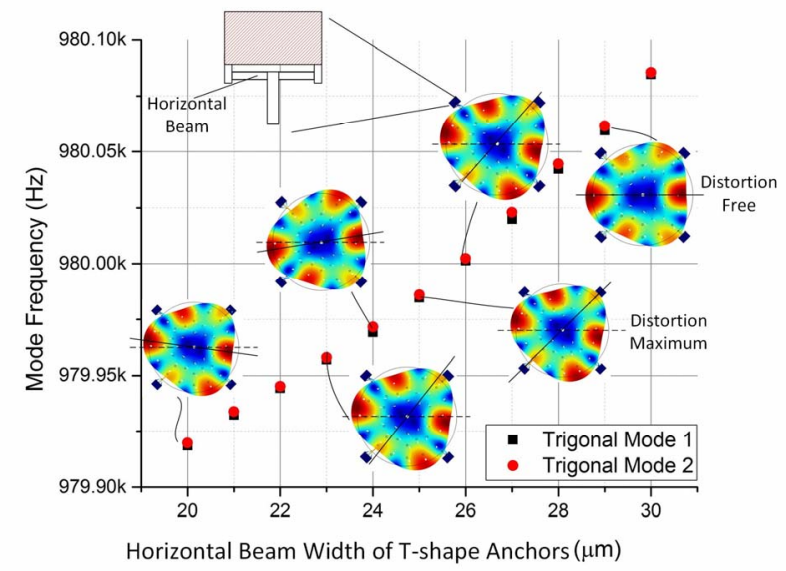

Figure 2: COMSOL simulation results of secondary elliptic degenerate modes resonance frequencies and mode-shape distortion (inset) with different horizontal beam width of Tshape Anchors.

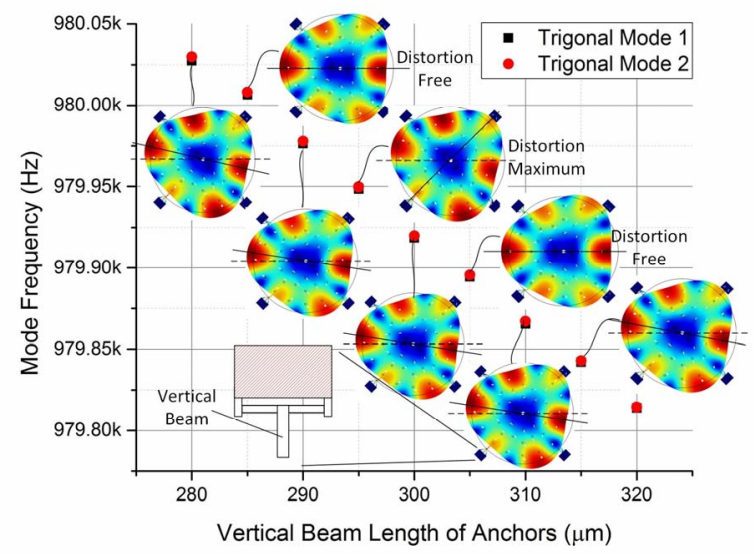

Figure 3: Numerical simulation results for the resonance frequencies of the trigonal modes and plots illustrating mode-shape distortion (inset) with different vertical beam length for T-shape anchors.

\section{C) Operational Electrode Design}

A group of capacitive electrodes surrounding the disk resonator are required for gyroscope operation. The locations of the electrodes are designed depending on the mode shape of the disk resonator. Generally, the drive and sense electrodes are located at the anti-nodes of driving mode and sensing mode, respectively. The control electrodes for resonance frequency tuning are located at the anti-nodes of the mode being tuned and the "decouple tuning" electrodes for mode-to-mode coupling cancellation are located where two degenerate modes have equivalent deflections. 


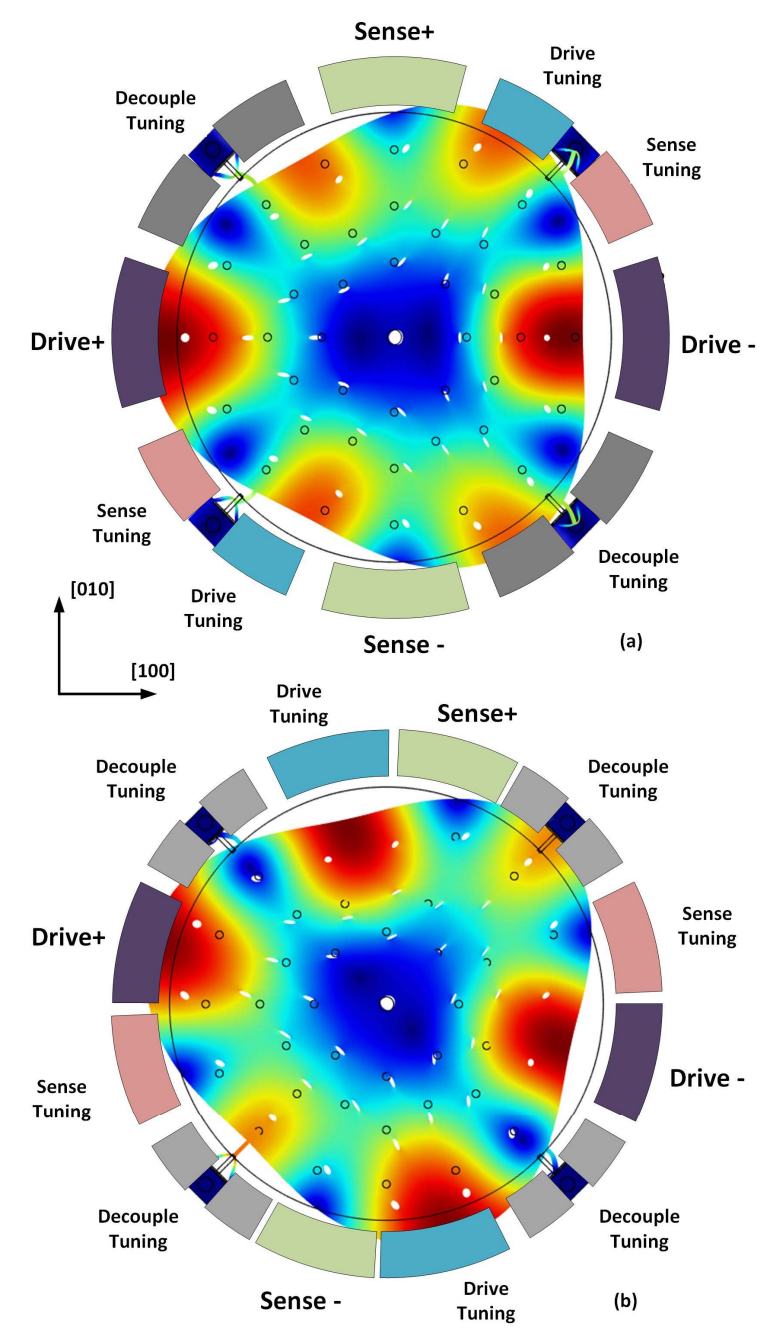

Figure 4: Electrode locations and functions for the edgeanchored disk gyroscope when the trigonal mode symmetric axis is aligned to the [100] direction (a) and the [110] direction (b).

As discussed, the mode shape of the edge-anchored disk resonator may vary with different $\mathrm{T}$-shape anchor dimensions and the electrodes locations need to be customized for each given mode shape. For brevity, only the electrode designs for 'distortion free' and 'distortion maximum' (refer to the insets of Figure 2 and Figure 3 ) cases are implemented as shown in Figure 4. In the case of 'distortion free' (see Figure 4 (a)), the drive and sense electrodes are centered at $90^{\circ}, 270^{\circ}$ and $0^{\circ}, 180^{\circ}$ with a radial angle of $45^{\circ}$, the frequency tuning electrodes for drive mode and sense mode are centered at $33.75^{\circ}, 213.75^{\circ}$ and $56.25^{\circ}$ and $236.25^{\circ}$ with a radial angle of $22.5^{\circ}$, and the decouple tuning electrodes are centered at $135^{\circ}$ and $315^{\circ}$ with a radial angle of $45^{\circ}$. In the case of 'distortion maximum' (see Figure 4(b)), the drive and sense electrodes are centered at $105^{\circ}, 285^{\circ}$ and $15^{\circ}, 195^{\circ}$ with a radial angle of $30^{\circ}$, the frequency tuning electrodes for drive mode and sense mode are centered at $165^{\circ}, 345^{\circ}$ and $75^{\circ}, 255^{\circ}$ with a radial angle of $30^{\circ}$, and the decouple tuning electrodes are centered at $135^{\circ}$ and $315^{\circ}$ with a radial angle of $30^{\circ}$. As the two modes for gyroscope operation are degenerate, the functions of drive/sense electrodes and drive tuning/sensing tuning electrodes can be switched.

\section{DEVICE FABRICATION AND CHARACTERIZATION}

To verify the above design methodology of edgeanchored disk gyroscopes, a batch of devices were designed and fabricated in a SOI process incorporating a wafer-level vacuum package. The key design parameters and simulation results are summarized in Table 1. The devices are then diced from the wafer, mounted and wire bonded to a LCC44 ceramic chip carrier as shown in Figure 5 .

Table 1: Summary of design parameters for the edgeanchored disk gyroscope

\begin{tabular}{|l|l|l|}
\hline \multicolumn{2}{|l|}{ Design Parameter } & Value \\
\hline Disk Diameter & $6 \mathrm{~mm}$ \\
\hline Device Thickness & $40 \mu \mathrm{m}$ \\
\hline $\begin{array}{l}\text { T-Shape } \\
\text { Suspensions }\end{array}$ & \begin{tabular}{l} 
Horizontal \\
Beam Width \\
\cline { 2 - 3 } \\
Horizontal \\
Beam Length
\end{tabular} & $25 \mu \mathrm{m}$ \\
\cline { 2 - 3 } & $\begin{array}{l}\text { Vertical } \\
\text { Beam Width }\end{array}$ & $60 \mu \mathrm{m}$ \\
\cline { 2 - 3 } & $\begin{array}{l}\text { Vertical } \\
\text { Beam Length }\end{array}$ & $295 \mu \mathrm{m}$ \\
\hline $\begin{array}{l}\text { Trigonal Mode Frequencies } \\
\text { (simulated) }\end{array}$ & $\begin{array}{l}979952.46 \mathrm{~Hz} / \\
979952.59 \mathrm{~Hz}\end{array}$ \\
\hline $\begin{array}{l}\text { Mode-shape Symmetric Axis } \\
\text { Orientation }\end{array}$ & $\begin{array}{l}<110>\text { (Distortion } \\
\text { Maximum Case) }\end{array}$ \\
\hline
\end{tabular}

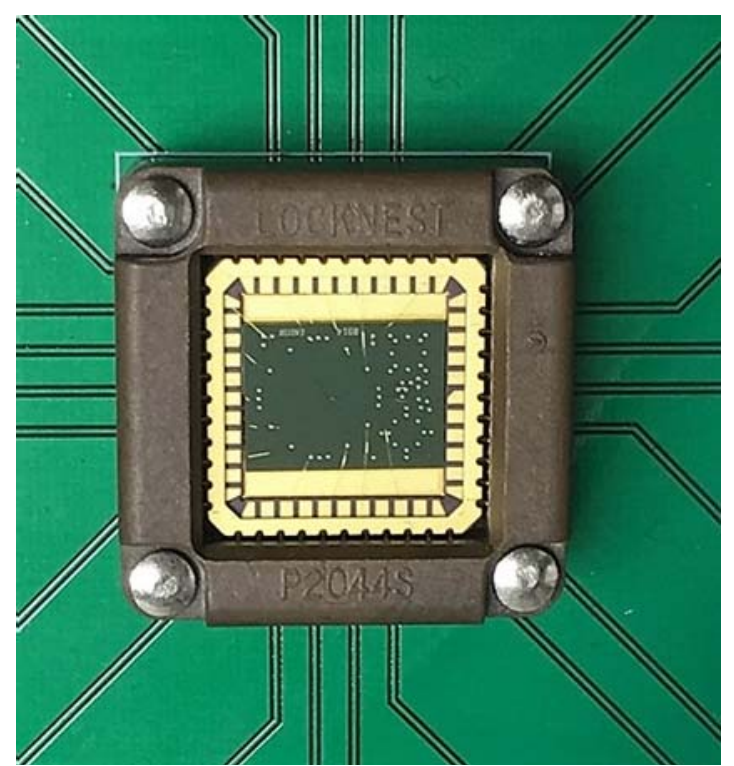

Figure 5. Picture of the edge-anchored disk gyroscope chip. 


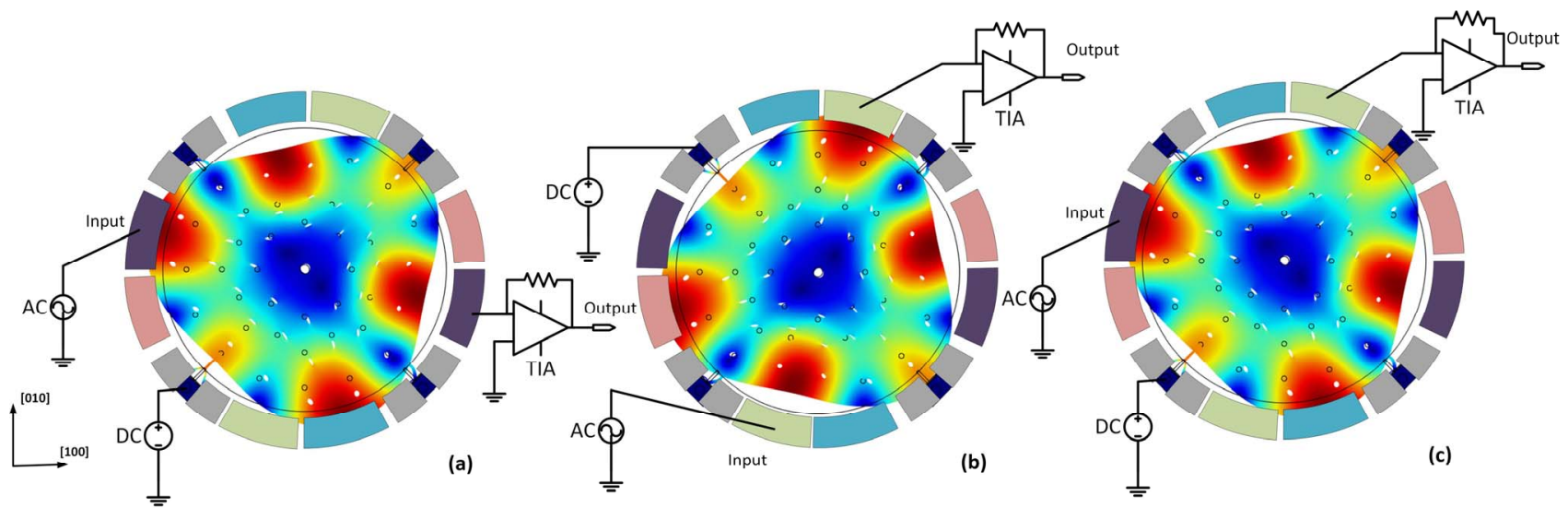

Figure 6: Setup Configuration for Direct Transmittance measurement of Mode 1(a) and Mode 2(b) and Cross-mode

Transmittance Measurement (c).

The test configuration for open-loop frequency characterization of BAW disk gyroscope is shown in Figure 6. A fixed DC bias voltage of $60 \mathrm{~V}$ is applied on the body of disk resonator and on the electrodes when they are not in use. The direct transmittance of two trigonal modes was measured when excited independently (see Figure 6(a) and (b)) and the cross-mode transmittance between two trigonal modes was measured where the signals pick-off from the electrodes orthogonal to the mode being excited (see Figure 6 (c)).

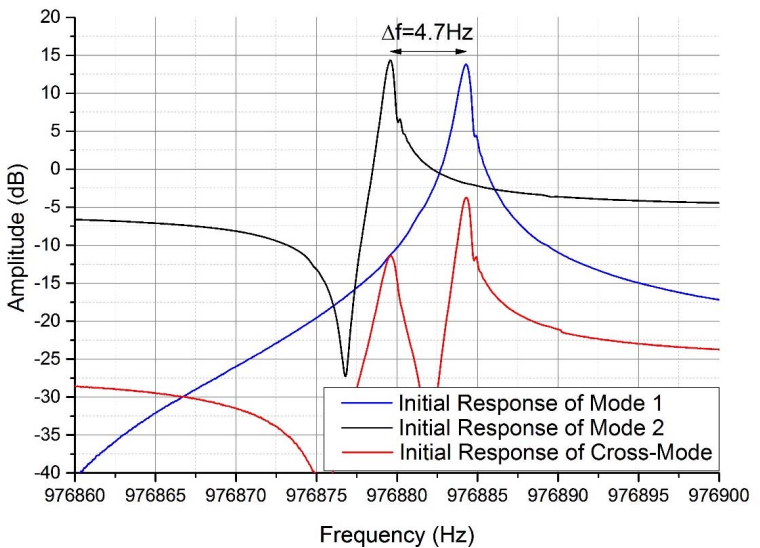

Figure 7: Measured initial frequency split of two trigonal modes of edge-anchored disk gyroscope.

As shown in Figure 7, the quality factor of the trigonal mode is over 1.5 million, the initial frequency split of the two trigonal modes is about $4.7 \mathrm{~Hz}$ and the cross-mode transmittance is 17.5 to $25 \mathrm{~dB}$ lower than the direct transmittance of the two modes itself. Due to the $4.7 \mathrm{~Hz}$ initial frequency mismatch shown for the device, it is possible to apply a DC tuning voltage of $-8 \mathrm{~V}$ on the tuning electrodes of Mode 1 to achieve full mode-matching (see Figure 8).

\section{SUMMARY}

This paper introduces a new resonator topology using T-shape edge anchors to implement a disk gyroscope. The design methodology is introduced, addressing the optimization of the dimensions of T-shape edge anchors to reduce the mode-distortion and to align the electrodes for gyroscope operation. A batch of devices was designed and fabricated in a SOI bonded wafer process and characterized to extract resonance frequencies, $\mathrm{Q}$ factor and initial mode frequency split of the degenerate mode pair for gyroscope operation. For a device with $4.7 \mathrm{~Hz}$ initial frequency split (4.81 ppm split), and Quality factor exceeding 1.5 million, mode-matching was demonstrated by electrostatic tuning. Ongoing work is addressing integration of the device with control electronics to implement a functional gyroscope.

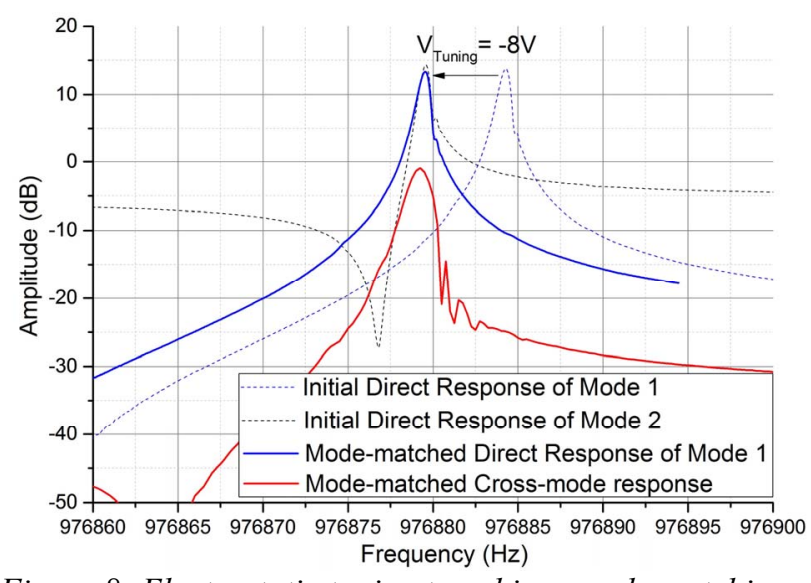

Figure 8: Electrostatic tuning to achieve mode matching.

\section{ACKNOWLEDGEMENTS}

Support from Innovate UK and the Natural Environment Research Council (NE/N012097/1) is acknowledged.

\section{REFERENCES}

[1] B. Vigna, It makes sense: How extreme analog and sensing will change the world, in: Technical Digest of the 2012 Solid-State Sensors, Actuators and Microsystems Workshop, Hilton Head, SC, 2012, pp. 58-65.

[2] C. Acar, A. Shkel, MEMS Vibratory Gyroscopes, Springer, New York, NY, 2009.

[3] H. Johari, F. Ayazi, Capacitive bulk acoustic wave silicon disk gyroscopes, in: Technical Digest of the IEEE International Electron Devices Meeting, San Francisco, CA, 2006, pp. 513-516.

[4] R. Mirjalili, H. Wen, D. E. Serrano, et al. Substratedecoupled silicon disk resonators having degenerate gyroscopic modes with $\mathrm{Q}$ in excess of 1-million, Proceedings of the 18th International Conference on Solid-State Sensors, Actuators and Microsystems (TRANSDUCERS), Anchorage, United States, 2015:15-18. 